\title{
Research on one Intelligent Open Online Course Based on Big Data Peng Zhang
}

Nanometer Information Department of Suzhou Industrial Park Institute of Services Outsourcing, Suzhou, 215123, China

zhangp@siso.edu.cn

Keywords: Open online course; Big data; Recommender-system; Neural network

\begin{abstract}
Open online courses integrate high-quality education resources and play active roles in the education system. This paper presents an intelligent open online course based on big data. This online course provides a variety of resources and massive exercises. Based on the recommender- system, the course recommends the most appropriate resources to each student. And based on the neural network, the course develops a suitable review plan for each student.
\end{abstract}

\section{Introduction}

In 2012, the Massachusetts Institute of Technology launched an open online class. Since then, Massive Open Online Courses (MOOCs) have been popular in the world. Open online courses integrate high-quality education resources and play active roles in the education system.

With the development of open online courses, high-quality teaching resources have accumulated more and more. In an online course, each knowledge point often corresponds to a number of videos, animations, coursewares, texts and other teaching resources, as well as a large number of exercises. The increase of types and quantities of resources enriched the teaching forms, but is also brings new problems: how do students choose resources? Excellent open online courses should guide students to learn the most effective teaching resources.

With the rapid development of new information technology, the informationization construction in colleges and universities is also facing great challenges. How to achieve intelligent online course is worth studying. Through the excavation and analysis of big data on campus, we can understand each student's living habits and interest in learning. Intelligent online courses can provide the most suitable teaching resources for each student, and can make the most effective learning plan for each student.

\section{Design of the Intelligent Online Course.}

The intelligent online course proposed in this paper needs to be integrated with big data. Its design is shown in Fig. 1.

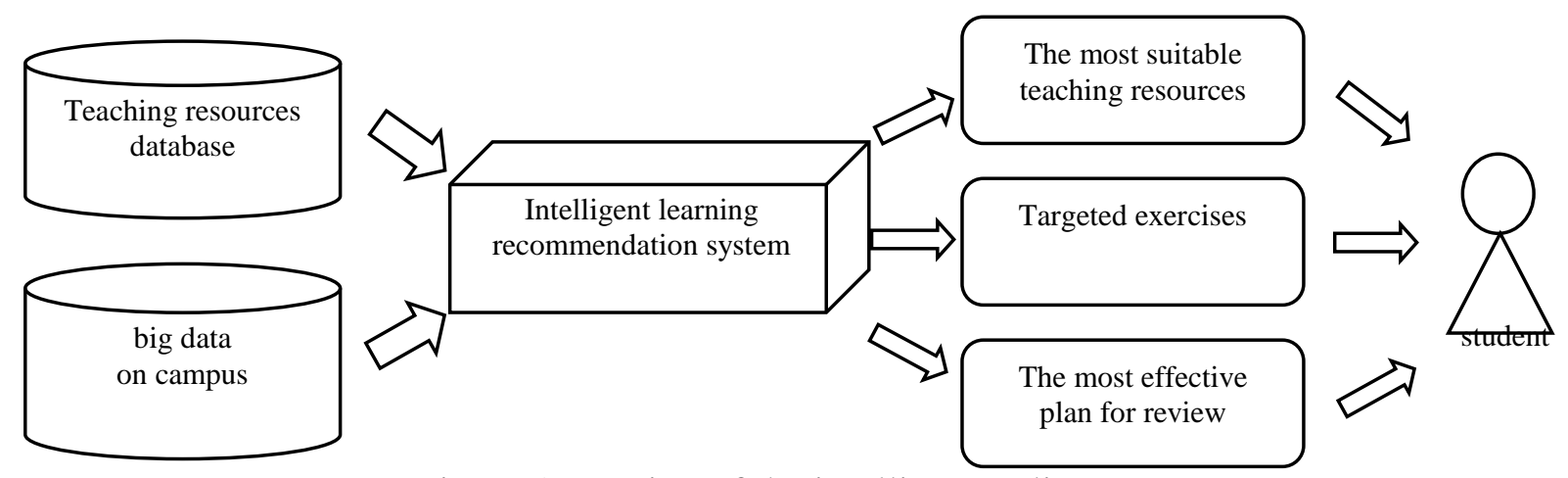

Figure 1. Design of the intelligent online course

In Fig. 1, the teaching resources database contains a wealth of teaching resources. According to the analysis of big data, intelligent learning recommendation system will filter the content of resources, 
and recommend the most suitable teaching resources to each student. In addition, the intelligent learning recommendation system will also develop a review plan for each student.

With the development of information technology, the big data on campus has accumulated over the years. How to exploit the valuable information contained in these data is a new challenge to the construction of information technology in Colleges and universities. Currently, the analysis of big data is mainly based on the Deep Neural Network (DNN) algorithm. In addition, the recommendersystem is also an effective way to solve the information overload. The intelligent learning recommendation system shown in Fig. 1 is based on neural network and recommender-system algorithm.

\section{How to Recommend the Most Suitable Teaching Resources to Students.}

The intelligent learning recommendation system in Figure1 adopts the User-Based Collaborative Filtering (UserCF) algorithm and recommends the most suitable teaching resources to each student. Before recommending resources, the system first finds other students who are similar to the target student. The following equation can be used to calculate the similarity about students' interest.

$$
\mathrm{S}_{\mathrm{ab}}=\frac{|\mathrm{N}(\mathrm{a}) \cap \mathrm{N}(\mathrm{b})|}{\sqrt{|\mathrm{N}(\mathrm{a})||\mathrm{N}(\mathrm{b})|}}
$$

In equation (1), $S_{a b}$ represents the similarity between Student A (SA) and Student B (SB). $N(a) \cap N(b)$ represents the set of resources that attract both SA and SB.

After obtaining the similarity of students' interest, we use the following equation to calculate the students' interest in a certain resource.

$$
X(a, i)=\sum_{b \in S(a, m) \cap N(i)} S_{a b} R_{b i}
$$

In equation (2), $X(a, i)$ represents the degree of SA's interest in Resource I (SI). $S(a, m)$ contains a set of users with similar interests to SA. $N(i)$ is a set of students interested in SI. $S_{a b}$ represents the similarity between SA and SB. $R_{b i}$ represents SB's interest in SI.

Using the above method, the system can know a student's interest in a certain resource. Then, the system can try to recommend suitable resources to this student.

\section{How to Recommend Targeted Exercises to Students.}

The intelligent learning recommendation system adopts the method of Item-Based Collaborative Filtering (ItemCF) and recommends targeted exercises to each student. Exercises have some relevance. For example, if a student has a problem with the exercise U, then he is likely to have problems with exercise $\mathrm{V}$. The following equation can be used to calculate the association of two exercises.

$$
Q_{u v}=\frac{|F(u) \cap F(v)|}{\sqrt{|F(u)||F(v)|}}
$$

In equation (3), $Q_{u v}$ represents the association of Exercise $\mathrm{U}(\mathrm{EU})$ and Exercise V (EV). $F(u)$ represents the number of students who have problems with EU. After getting the association of the exercises, we can predict the importance of EU to Student A (SA) by the following equation.

$$
Y(a, v)=\sum_{u \in F(u) \cap Q(v, K)} Q_{u v} T_{a u}
$$


In equation (4), $Q(v, K)$ represents a set of exercises associated with $\mathrm{EV}, T_{a u}$ indicates the importance of EU to SA.

Using the above method, the system can understand the importance of an exercise to a student. Then, the system can try to recommend targeted exercises to this student. Of course, if the system is only recommended targeted exercises, which may reduce the confidence of students. Therefore, the system should mark the importance of all exercises. Then, students choose suitable exercises by themselves.

\section{How to Make Review Plans for Students.}

H.Ebbinghaus, a German psychologist, found that forgetting began immediately after learning, and the process of forgetting was not uniform. His experimental results are plotted to describe the forgotten process. The forgetting curve is shown in Fig. 2.

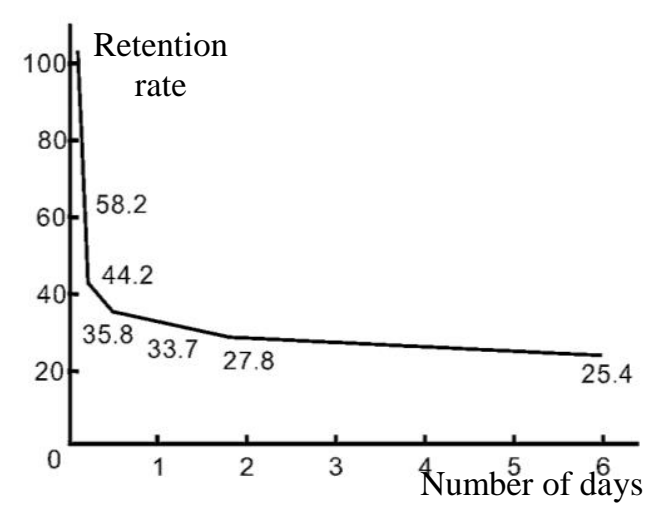

Figure 2. The forgetting curve

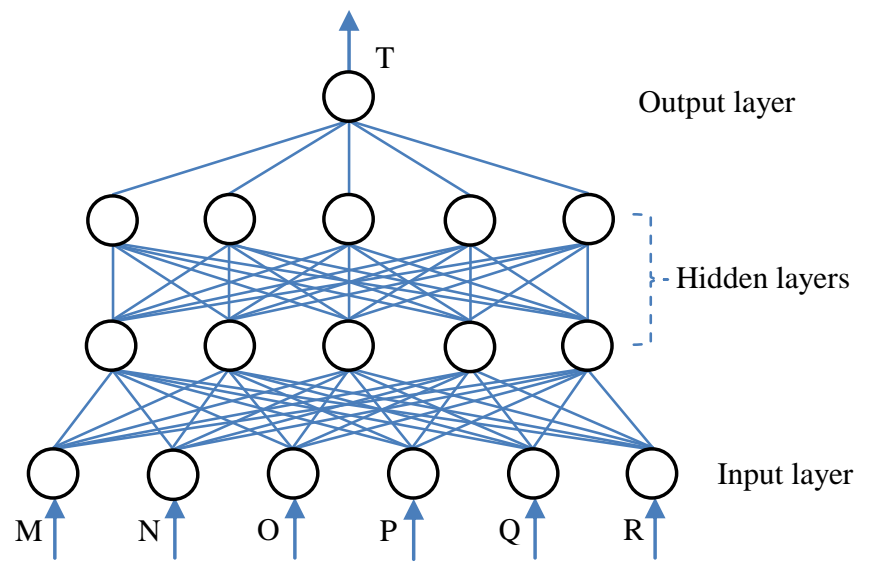

Figure 3. Network structure

After careful study, the input information has become a short-term memory. But if you do not review in time, the information will be forgotten. And after a timely review, these short-term memory will become a long-term memory.

In addition, we also believe that each student's memory should be different. The effect of each review should be related to the student's memory, the number of reviews, the time interval, the difficulty of the knowledge and so on. Because the students' memory is hard to describe in numerical terms, so we use the IQ, the average score in the past and the online learning activity to replace it. The IQ was obtained through online Q \& A tests. We use the following equation to calculate the number of days that a student can keep the memory after each review.

$$
T=\sqrt{\frac{\mathrm{aM}^{2}+\mathrm{bN}^{2}+\mathrm{cO}^{2}+\mathrm{dP}^{2}+\mathrm{eQ}^{2}+\mathrm{fR}^{2}}{6}}
$$

In Equation (5), $\mathrm{T}$ indicates the number of days that the student can keep memory. After $\mathrm{T}$ days, the system will arrange for students to review again. $M$ indicates the number of times the student has reviewed. $\mathrm{N}$ indicates the time interval between this review and the last review. $\mathrm{O}$ indicates the student's IQ. P represents the average score of the student in the past. Q represents the student's learning activity. $\mathrm{R}$ indicates the difficulty of this knowledge point. The $\mathrm{a}, \mathrm{b}, \mathrm{c}, \mathrm{d}$, e and $\mathrm{f}$ are the weights of the parameters, and their initial values are $2,1.5,0.05,0.05,0.05$ and 0.05 . These weights can be corrected by analyzing the big data on the campus.

At present, the analysis of big data mainly uses BP neural network (BPNN), convolutional neural network (CNN), recurrent neural network (RNN) and LSTM neural network (LSTM). CNN is suitable for the identification of images and voices. RNN is suitable for language modeling. LMSL is suitable for the recognition of voices, pictures and natural languages. 
The intelligent recommendation system proposed in this paper uses BPNN to analyze the big data on campus. BPNN is a Feedforward Neural Network, and its network structure is shown in Fig. 3. There are 6 nodes in the input layer of the network, but only 1 node in the output layer. Because the number of BPNN's hidden layers should not be too much, so there are only 2 hidden layers. Each hidden layer has 5 nodes. The activation function of the neural network is "sigmoid".

At each review, the system will test the student, and adjust the neural network based on the test results. If the student's test results are ideal, the network uses the following equation as the objective function. Where the initial value of $\beta$ is 0.2 .

$$
E_{\text {d }}=\frac{1}{2}(\beta T+2)^{2}
$$

If the student's test results are not good, the network uses the following equation as the objective function. The function min can get the minimum value.

$$
E_{d}=-\min \left(T-1, \frac{1}{2}(\beta T+2)^{2}\right)
$$

We also optimize the weights of the network through genetic algorithms. The weights are encoded to produce chromosomes. Then, the chromosomes are crossed, matched, and mutated, so that the network evolves continuously.

\section{Analysis of Practical Data.}

3 years ago, we set up two open online courses of "C language programming". One is intelligent and the other is traditional. We divide the students into two groups: $\mathrm{M}$ and N. Students in the group $\mathrm{M}$ studied the intelligent open online course, while students in the group $\mathrm{N}$ studied the traditional open online course.

There are 6 semesters in 3 years. During this period, 716 students were enrolled in the group M, and 734 students were enrolled in the group N. The average score of each semester of the course is shown in Fig. 4. Obviously, in each semester, the average score of the group $M$ was higher than that of the group N. Moreover, with the continuous training of neural networks, the system becomes more and more intelligent. The average scores of $\mathrm{M}$ students are also increasing.

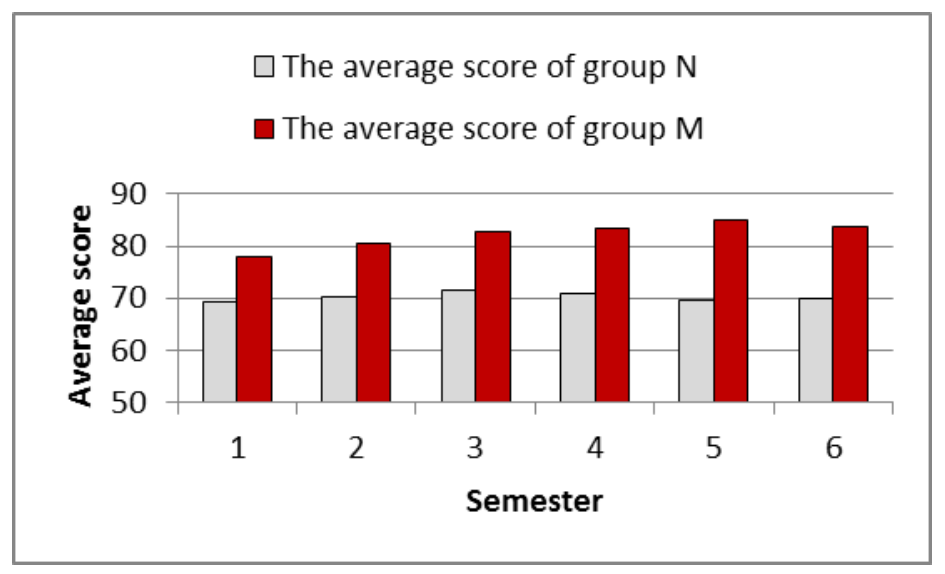

Figure 4. The average scores

"Java Language Programming (JAVA)" is similar to "C Language Programming (C)". Students in the group $\mathrm{M}$ have also studied the traditional open online course of "JAVA". We randomly selected 30 samples from the group M. Their scores of two courses are shown in Fig. 5. Obviously, for most students, scores of "C" are higher than those of "JAVA". 


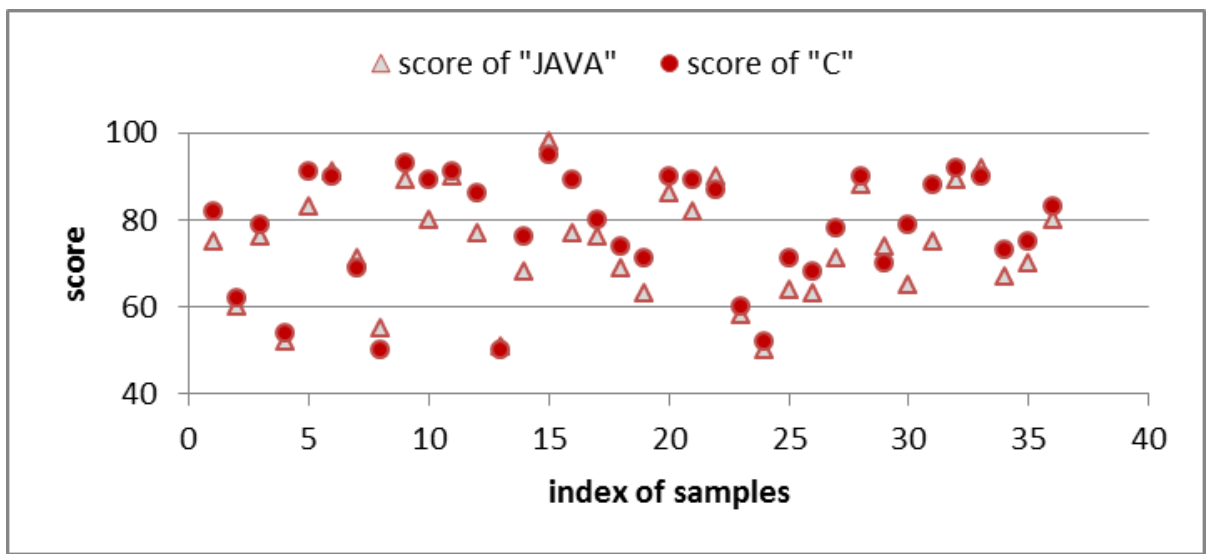

Figure 5. Students' scores of two similar courses

We choose a class (class A) from the $\mathrm{M}$ group, and choose another class (class B) from the $\mathrm{N}$ group. Except for "C", the average scores of other courses in the two classes are almost the same. The scores of "C" in the two classes are shown in Fig. 7. Obviously, the scores of students from class A are generally higher.

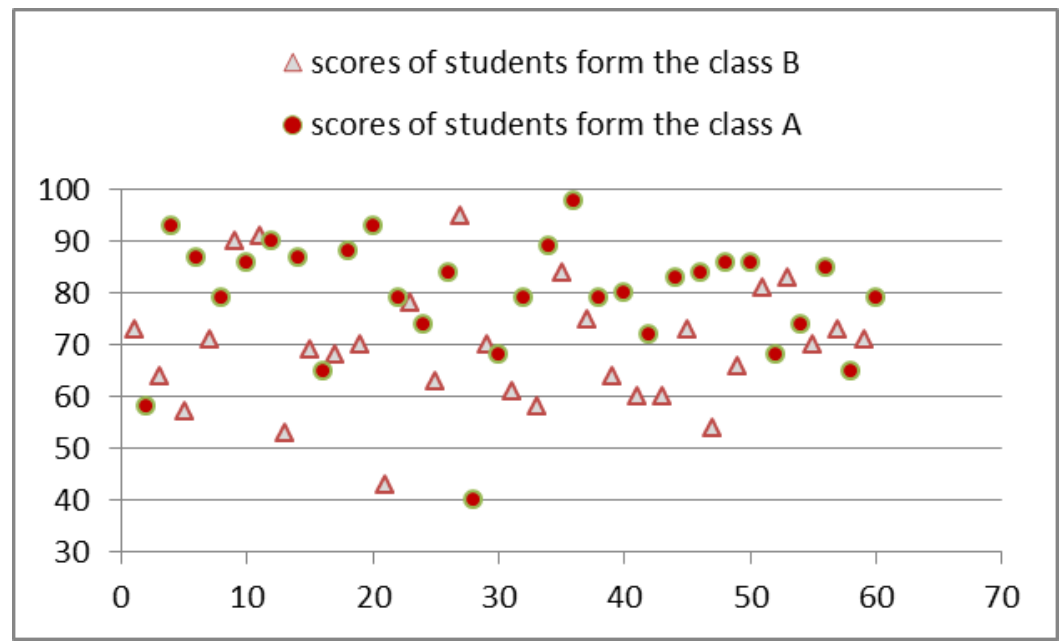

Figure 6. Students' scores of two similar classes

After learning the "C", teachers also evaluated the students' practical ability. The results of the evaluations in the two classes are shown in Fig. 7. Obviously, the class A is better.

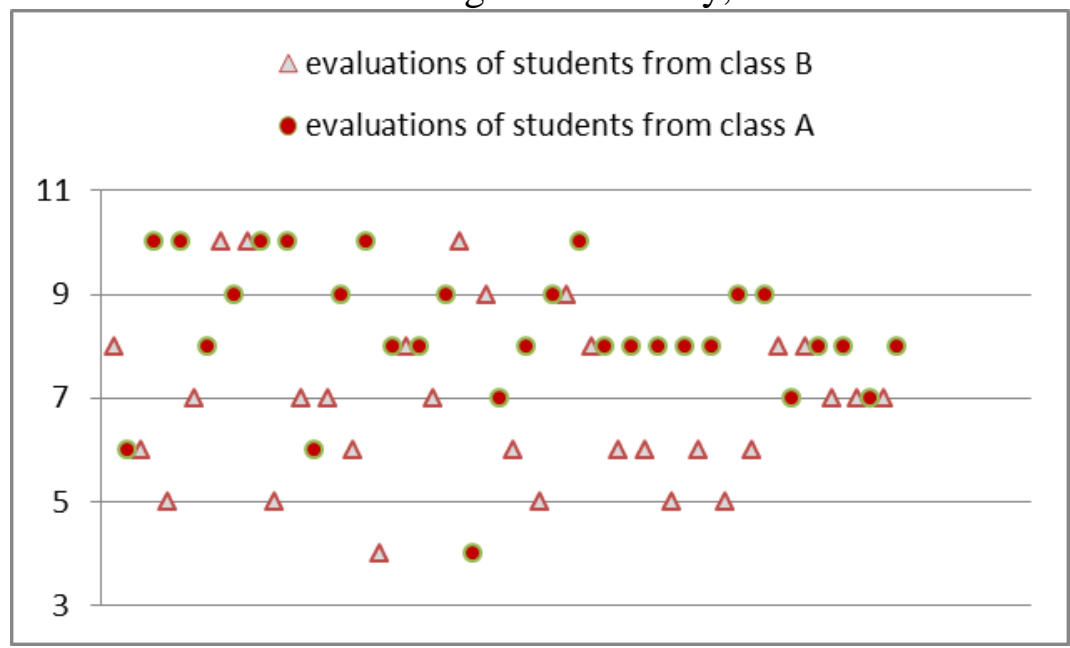

Figure 7. Evaluations of two similar classes 


\section{Summary}

The practice data demonstrates the advantages of the intelligent online open course. The intelligent online course recommends the most suitable teaching resources to each student. Compared with the traditional online courses, the intelligent online course is more suitable for modern education system.

\section{Acknowledgements}

This research was financially supported by the Jiangsu Province Department of Education Reform Project (No. 2015JSJG396) and the Jiangsu Province Science education project in 13th Five-Year. (No. C-c/2016/03/14).

\section{References}

[1] Stacked denoising autoencoders: Learning Useful Representations in a Deep Network with a Local Denoising Criterion. Vincent, Pascal, Larochelle, Hugo, Lajoie, Isabelle, Bengio, Yoshua, Manzagol, Pierre-Antoine. Journal of Machine Learning Research. 2010

[2] Imagenet classification with deep convolutional neural networks. A. Krizhevsky,I. Sutskever, G. Hinton. NIPS. 2012

[3] Imagenet classification with deep convolutional neural networks. A. Krizhevsky,I. Sutskever,G. Hinton. NIPS. 2012

[4] Caffe: convolutional architecture for fast feature embedding. JIA Y, SHELHAMER E, DONAHUE J,et al. Proceedings of the ACM International Conference on Multimedia . 2014

[5] Long short-term memory based recurrent neural network architectures for large vocabulary speech recognition. Sak H,Senior A, Beaufays F. . 2014

[6] Sequence to sequence learning with neural networks. Sutskever I,Vinyals 0,Le QVV. Advances in Neural Information Processing Systems. 2014

[7] Generating sequences with recurrent neural networks. Graves A. 2013

[8] Hybrid speech recognition with deep bidirectional LSTM. Graves A, Jaitly N, Mohamed A. Proc of 2013 IEEE Workshop on Automatic Speech Recognition and Understanding (ASRU). 2013

[9] LSTM-based early recognition of motion patterns. Weber M, Liwicki M,Stricker D,et al. Proc of the 22nd Int Conf on Pattern Recognition (ICPR2014) . 2014

[10] Improved semantic representations from tree-structured long short-term memory networks. Tai K S,Socher R,Manning C D. . 2015

[11]Exploring Spatiotemporal and Social Network Factors in Community Response to a Major Flood Disaster[J] . Kathleen Stewart,Jennifer L. Glanville, David A. Bennett. The Professional Geographer . 2014 (3)

[12]Mining geographic-temporal-semantic patterns in trajectories for location prediction[J] . Josh Jia-Ching Ying,Wang-Chien Lee, Vincent S. Tseng. ACM Transactions on Intelligent Systems and Technology (TIST) . 2014 (1)

[13]Context-Aware Recommender Systems for Learning: A Survey and Future Challenges. Katrien Verbert,Nikos Manouselis,Xavier Ochoa. IEEE TRANSACTIONS ON LEARNING TECHNOLOGIES . 2012

[14]Human mobility, social ties, and link prediction. Dashun Wang, Dino Pedreschi, Chaoming Song,Fosca Giannotti, Albert-Laszlo Barabasi. Proceedings of the 17th ACM SIGKDD international conference on Knowledge discovery and data mining . 2011

[15] What you are is when you are:the temporal dimension of feature types in location-based social networks. Ye M,Janowicz K, Mulligann C, et al. Proceedings of the 19th ACM SIGSPATIAL International Conference on Advances in Geographic Information Systems . 2011

[16]Mining significant semantic locations from GPS data. Xin Cao, Gao Cong, Christian S. Jensen. Proceedings of the VLDB Endowment . 2010

[17]Mining User Mobility Features for Next Place Prediction in Location-Based Services. Noulas A,Scellato S, Lathia N, et al. ICDM . 2012 
[18]"City-scale traffic simulation from digital footprints.". Mc Ardle, Gavin,Aonghus Lawlor, Eoghan Furey, Alexei Pozdnoukhov. Proceedings of the ACM SIGKDD International Workshop on Urban Computing . 2012

[19]Cross-region collaborative filtering for new point-of-interest recommendation. Zheng Ning,Jin Xiaoming, Li Lianghao. Proceedings of the 22ndInternational Conference on World Wide Web Companion . 2013

[20] Simulation-based learning: The learning-forgetting-relearning process and impact of learning history[J] . Lior Davidovitch, Avi Parush, Avy Shtub. Computers \& Education . 2006 (3) 